\title{
Dinâmica populacional de minador dos citros em pomares de laranja De Russas no semiárido cearense
}

\author{
Francisco Leandro Costa Loureiro ${ }^{1}$, Raimundo Ivan Remígio Silva ${ }^{2}$, \\ Paulo Henrique Melo Gadelha ${ }^{1}$, Kássio Ewerton Santos Sombra ${ }^{1 *}$, Orlando Sampaio Passos ${ }^{3}$, \\ Débora Costa Bastos ${ }^{4}$, Renata Damasceno Moura ${ }^{5} \&$ Cleilson do Nascimento Uchôa ${ }^{2}$
}

\section{RESUMO}

As larvas de Phyllocnistis citrella Stainton (Lepidoptera: Gracillariidae) ocasionam injúrias em diferentes plantas do gênero Citrus (Rutaceae), reduzindo taxa fotossintética e desenvolvimento de novas brotações, ocasionando seca e queda das folhas afetadas, além de facilitar a penetração de Xanthomonas citri subsp. citri, bactéria causadora do cancro cítrico. Diante disto, conhecer a variação sazonal da praga pode otimizar seu manejo. O objetivo do trabalho foi caracterizar a dinâmica populacional das larvas em plantas de laranja De Russas [Citrus sinensis (L.) Osbeck] ao longo de 12 meses. Realizaram-se amostragens quinzenalmente, registrando-se o total de brotações contendo minas ou larvas, número de folhas com larvas por brotação, além do número de larvas, minas e pupas por folha. O desenvolvimento das laranjeiras foi registrado por avaliações biométricas, enquanto dados meteorológicos foram utilizados para gerar um gráfico da incidência em função da condição climática. Constatou-se que a oferta de alimento (novas brotações) ao longo do ano, apesar de influenciar na ocorrência do minador, não foi o fator mais significativo, verificando-se que a densidade populacional variou principalmente devido a temperatura e umidade. Os maiores índices de larvas e pupas foram registrados nos primeiros meses, resultando em $43 \%$ de brotações atacadas e até 3 minas por folha. As condições que propiciaram maiores densidades populacionais foram temperaturas próximas à $26^{\circ} \mathrm{C}$, precipitações de $60 \mathrm{~mm}$ e umidade relativa de $60 \%$. O que na prática pode otimizar o manejo integrado de pragas, permitindo a adoção de técnicas preventivas para manter as populações de minador dos citros abaixo do nível de controle.

Termos de indexação: amostragem, citricultura, manejo de pragas, Phyllocnistis citrella.

\section{Population dynamics of citrus leafminer in De Russas orange orchards in semiarid region of Ceará}

\section{SUMMARY}

The larvae of Phyllocnistis citrella Stainton (Lepidoptera: Gracillariidae) cause insults in different plants the genus 'citrus' (Rutaceae), reducing photosynthetic rate and development new shoots, causing drought and fall of the affected leaves, besides facilitating the penetration of Xanthomonas citri subsp. citri, which causes citrus canker. In view of this, knowing the seasonal variation the

\footnotetext{
${ }^{1}$ Programa de Pós-graduação em Fitotecnia, Universidade Federal Rural do Semi-Árido - UFERSA, Mossoró, RN, Brasil

${ }^{2}$ Instituto Federal de Educação, Ciência e Tecnologia do Ceará - IFCE, Limoeiro do Norte, CE, Brasil

${ }^{3}$ Embrapa Mandioca e Fruticultura, Cruz das Almas, BA, Brasil

${ }^{4}$ Embrapa Semiárido, Petrolina, PE, Brasil

${ }^{5}$ Agência de Defesa Agropecuária do Estado do Ceará - ADAGRI, Limoeiro do Norte, CE, Brasil

Autor correspondente: Kássio Ewerton Santos Sombra, Programa de Pós-graduação em Fitotecnia, Universidade Federal Rural do SemiÁrido - UFERSA, Av. Francisco Mota, 572, Bairro Costa e Silva, CEP 59625-900, Mossoró, RN, Brasil. E-mail: kassiosombra@gmail.com
} 
pest can optimize its management. The objective this work was to characterize the population dynamics of larvae in De Russas orange plants [Citrus sinensis (L.) Osbeck] over 12 months. Samples were collected biweekly, recording the total number of shoots containing mines or larvae, number of leaves with larvae, number of larvae, mines and pupae per leaf. The development of orange trees was recorded by biometric evaluations, while meteorological data were used to generate a graph the incidence as a function of the climatic condition. It was found that the supply of food (new shoots) throughout the year, despite influencing the occurrence of the miner, was not the most significant factor, and it was verified that the population density varied mainly due to temperature and humidity. The highest rates of larvae and pupae were recorded in the first months, resulting in $43 \%$ of sprouts attacked and up to 3 mines per leaf. The conditions that provided higher population densities were temperatures close to $26^{\circ} \mathrm{C}$, precipitations of $60 \mathrm{~mm}$ and relative humidity of $60 \%$. This in practice can optimize the integrated pest management, allowing the adoption of preventive techniques to keep citrus miner populations below the control level.

Index terms: sampling, citriculture, pest management, Phyllocnistis citrella.

\section{INTRODUÇÃO}

Phyllocnistis citrella Stainton (Lepidoptera: Gracillariidae) é um microlepidóptero originário do sudeste asiático, conhecido como minador dos citros devido as injúrias ocasionadas pelo seu estágio larval. As larvas abrem galerias em forma de serpentina à medida que se alimentam do mesófilo foliar de plantas do gênero 'Citrus', sendo praga constante em praticamente todos os países produtores, citando-se alguns como Austrália, África do Sul, Estados Unidos e países da América Central (Cônsoli et al., 1996; Mattos Junior et al., 2005; Brito et al., 2017). No Brasil, o primeiro registro do minador dos citros ocorreu em pomares cítricos de Iracemápolis, SP, em março de 1996. Desde então, o microlepidóptero se disseminou nos polos citrícolas nacionais, ocasionando sérios prejuízos a cadeia produtiva (Cônsoli et al., 1996; Yamamoto, 2008; Gravena, 2011).

Os prejuízos diretos são decorrentes da diminuição da superfície foliar, do engruvinhamento e da queda de folhas danificadas, que reduzem a eficiência fotossintética e limitam o desenvolvimento de novas brotações, afetando a produção e a qualidade dos frutos cítricos (Gravena, 2005; Arshad et al., 2018). As injurias podem refletir em perdas de até $60 \%$ no peso dos frutos, e de até $50 \%$ da produtividade média (Gravena, 2005; Yamamoto, 2008). No entanto, os principais prejuízos são decorrentes de associações com fitopatógenos, como a bactéria causadora do cancro cítrico, Xanthomonas citri subsp. citri, que utiliza as injurias como porta de entrada, facilitando a penetração e infecção (Rossetti, 2001; Mattos Junior et al., 2005). A associação entre o minador e o cancro cítrico já era evidente após o primeiro registro do microlepidóptero no Brasil, quando se constatou, por exemplo, aumento de dez vezes no número de áreas infectadas pela doença em pomares do Estado de São Paulo (Parra et al., 2004; Gravena, 2011).

O controle químico ainda é o método mais utilizado no manejo do minador dos citros, geralmente feito por meio de pulverizações foliares, utilizando produtos à base de Abamectina e óleo mineral (De Morais et al., 2016; Qureshi et al., 2017). No entanto, tem crescido a adoção de métodos alternativos, que apresentem eficácia no controle do micro lepidóptero, más também segurança aos produtores, consumidores e meio ambiente; e seletividade a organismos não-alvo, como polinizadores e inimigos naturais; destacando-se o controle biológico utilizando Ageniaspis citricola Logvinovskaya (Hymenoptera: Encyrtidae) (Cônsoli et al., 1996; Yamamoto, 2008). A microvespa parasita o ovo e o primeiro estágio da larva, e sua presença nos pomares pode resultar em parasitismos de até $76 \%$, reduzindo significativamente a população do minador dos citros, e com isso, as contaminações pela bactéria do cancro cítrico (Gravena, 2011; Paiva \& Yamamoto, 2015; De Morais et al., 2016).

Porém, a eficiência do controle biológico, e de outros métodos, está diretamente ligada a tecnologia de liberação/ aplicação e a tomada de decisão no manejo integrado de pragas (MIP), que deve considerar o momento e a técnica mais adequada à densidade populacional, e sua capacidade de ocasionar danos ou injúrias, além das condições econômicas e ambientais nos pomares de laranja doce [Citrus sinensis (L.) Osbeck] (Rutaceae), tornando-se fundamental conhecer a dinâmica e a sazonalidade do micro lepidóptero, a fim de otimizar o seu controle (Parra et al., 2004; Gravena, 2011, 2005; Yamamoto, 2008).

Diante do exposto, o trabalho teve como objetivo avaliar a ocorrência e a dinâmica populacional do minador dos citros ( $P$. citrella) em pomares de laranja De Russas [Citrus sinensis (L.) Osbeck] no semiárido do Ceará. 


\section{MATERIAL E MÉTODOS}

O estudo foi desenvolvido entre janeiro e dezembro de 2016, em pomares de laranja doce, localizados em pequenas propriedades de agricultura familiar, na zona rural do Município de Russas, CE, Brasil.

Russas é um município localizado na microrregião do Baixo Jaguaribe, com área circunscrita às coordenadas geográficas $4^{\circ} 56^{\prime} 24^{\prime \prime} \mathrm{S}$ e $37^{\circ} 58^{\prime} 33^{\prime \prime} \mathrm{W}$, com altitude de 20,51 m, e clima classificado como semiárido (seco e muito quente), do tipo BSw'h' (Köppen) (Alvares et al., 2013). A microrregião insere-se no bioma caatinga, e apresenta entre outras características edafoclimáticas: temperatura média anual de $27,1^{\circ} \mathrm{C}$, oscilando entre 22,3 ${ }^{\circ} \mathrm{C}$ de temperatura média mínima e $34,8{ }^{\circ} \mathrm{C}$ de máxima; umidade relativa (UR) do ar entre $44 \%$ e $85 \%$; e um regime pluviométrico médio de $750 \mathrm{~mm}$, variando entre 548 e $992 \mathrm{~mm}$, distribuídos irregularmente em duas estações, uma seca que vai geralmente de julho a dezembro (verão), e uma chuvosa, de janeiro a junho (inverno) (INMET, 2018), com ocorrência de solos aluviais e vegetação nativa formada predominantemente por carnaubeiras, Copernicia prunifera Mill. H. E. Moore (Arecaceae) (Sombra et al., 2016, 2018a, 2018c).

Adotou-se o delineamento em blocos casualizados (DBC), analisando a ocorrência e a dinâmica populacional do lepidóptero-praga ao longo dos 12 meses, utilizando seis repetições (pomares) e parcela útil de 10 laranjeiras, sorteadas aleatoriamente em cada bloco (repetição).

Os pomares tinham cerca de 3 anos de idade, e apresentavam em média 80 plantas, distribuídas em espaçamento de $5 \times 2 \mathrm{~m}$, numa área de aproximadamente 0,1 hectare. O manejo consistia de adubação orgânica à base de esterco e restos vegetais (fundação e cobertura anual), a demanda hídrica da cultura era suprida por irrigação (1/3 das propriedades por superfície, e $2 / 3$ por irrigação localizada), enquanto os tratos culturais consistiam apenas de capinas ou roços regulares, remoção de brotos 'ladrões', coroamento (bacia de capitação de água) e adição de cobertura morta em superfície (palha de carnaúba triturada) (Sombra et al., 2018a, 2018c).

As amostragens foram realizadas quinzenalmente, sorteando-se as plantas úteis com base no croqui de implantação, e identificando-as em campo com fitas de seda para facilitar a localização. As plantas foram inspecionadas visualmente, dividindo-se a copa em quatro quadrantes (Norte, Sul, Leste e Oeste), para facilitar a leitura e interpretação da ocorrência e intensidade de infestação do minador dos citros (Paleari et al., 2001; Greve \& Redaelli, 2006). Em cada quadrante, registrou-se o número total de novas brotações, que foram distinguidas por apresentar coloração verde clara, tecido tenro e a folha basal medindo até $3 \mathrm{~cm}$, e diferenciadas pela presença ou ausência de larvas ou minas. Logo após, selecionou-se uma brotação aleatoriamente por quadrante, registrando-se o número de folhas com larvas/minas, além do número de minas, de larvas e de pupas por folha (Paleari et al., 2001; Jahnke et al., 2008; Keathley et al., 2015).

As médias foram comparadas pelo teste de Scott Knott $(p \leq 0,05)$ nos casos de diferença significativa, utilizando o software estatístico Sisvar ${ }^{\circledR}$ (Ferreira, 2014).

O desenvolvimento vegetativo das laranjeiras De Russas foi determinado por meio de três avaliações biométricas, distribuídas nos meses de janeiro (mês 0), junho (mês 6) e dezembro (mês 12). As variáveis consistiram de altura (H), medida do solo ao ápice da planta com auxílio de trena métrica graduada em centímetros, assim como o diâmetro de copa entre linhas e entre plantas (Dr e Dl) (Bastos et al., 2017a, 2017b; Sombra et al., 2017, 2018b). Calculando-se o volume de copa $\left(\mathrm{m}^{3}\right)$, utilizando a fórmula de Turrell (1946), descrita a seguir:

$\mathrm{V}=\left(\frac{\pi}{6}\right)$. H. DI. Dr

onde: $\mathrm{V}=$ volume de copa; $\mathrm{H}=$ altura; $\mathrm{DI}=$ diâmetro de copa no sentido entre linha; $\mathrm{Dr}=$ diâmetro de copa no sentido entre planta.

As condições climáticas foram analisadas a partir das temperaturas máxima, mínima e média, da precipitação e da umidade relativa do ar, obtidas pela estação meteorológica automática do Instituto Nacional de Meteorologia INMET, localizada a uma distância média de $24 \mathrm{~km}$ dos pomares avaliados, no Município de Jaguaruana. Para fins estatísticos, utilizou-se a média dos quinze dias que antecederam cada amostragem.

As possíveis relações entre o desenvolvimento vegetativo, as condições climáticas e as densidades populacionais do minador dos citros, foram submetidas a análise de regressão $(p \leq 0,01)$. Enquanto os valores de temperatura e umidade relativa foram utilizados na elaboração do gráfico de superfície de resposta, da intensidade de infestação em função das condições climáticas, utilizando o software estatístico Sisvar ${ }^{\circledR}$ (Ferreira, 2014).

\section{RESULTADOS E DISCUSSÃO}

A dinâmica populacional do minador dos citros (P. citrella) variou ao longo dos 12 meses de avaliação, constatando-se diferença significativa entre as variáveis 
analisadas $(p<0,01)$ (Tabela 1$)$, com destaque a intensidade de infestação (II), fundamental a tomada de decisão no manejo integrado de pragas (MIP), além do número de minas (MF), larvas (LF) e pupas por folhas (PF) de laranjeiras De Russas, que permitem conhecer a fase predominante no momento de cada leitura, e consequentemente, avaliar quais táticas ou medidas podem ser empregadas no manejo, ou no controle, do minador dos citros.

O levantamento foi realizado apenas em novas brotações, devido à preferência de oviposição das fêmeas adultas do minador dos citros por folhas e ramos tenros (Garijo \& García, 1994; Peña \& Schaffer, 1997; Fundecitrus, 2019). Observando-se as maiores densidades populacionais nos primeiros meses do ano, com pico de intensidade de infestação (II) entre janeiro e fevereiro (Figura 1), associando-se principalmente à disponibilidade de recursos/hospedeiro, que por sua vez foi influenciada pelas condições meteorológicas favoráveis, observadas no primeiro semestre do ano, durante a estação de inverno no semiárido cearense. No entanto no segundo semestre, a intensidade de infestação média foi de $21,31 \%$, com leve pico no mês de outubro, com 29,44\% (Figura 1), acima do nível de controle proposto por alguns autores (Cônsoli et al., 1996; Yamamoto, 2008; Gravena, 2011; Fundecitrus, 2019). Por exemplo, Pereira (2008) recomendou o controle do minador dos citros com valores acima de $10 \%$ em pomares jovens, enquanto em pomares adultos, $\mathrm{o}$ autor recomendou que controle fosse empregado quando o nível atingisse $40 \%$.

Greve \& Redaelli (2006) avaliaram a variação sazonal dos estágios imaturos do minador dos citros em dois pomares de laranjeira doce cv. Valência no Município de Montenegro, Rio Grande do Sul, e evidenciaram que o manejo do minador geralmente concentra-se em uma época do ano, quando as condições favoráveis permitem o estabelecimento e o incremento populacional do micro lepidóptero, e que diante disto, o produtor poderia monitorar os fatores climáticos, como maneira de antecipar e reduzir a intensidade de infestação inicial, e consequentemente, elevar a eficiência do MIP (Greve \& Redaelli, 2006; Gravena, 2005).

As maiores densidades resultaram em maior número de brotações e de folhas com minas, de larvas e de pupas por folha, nos meses de janeiro e fevereiro (Tabela 2), persistindo com valores intermediários até o final do primeiro semestre. Entretanto, no segundo os valores caíram consideravelmente, oscilando entre 0,3 e 0,6 folhas com minas por brotação (Tabela 2).

Nos meses de julho a dezembro a atividade do minador diminuiu (Tabela 2), possivelmente influenciada pela menor número de novas brotações (Figura 2) e condições climáticas, assemelhando-se aos resultados obtidos por Greve \& Redaelli (2006), que observaram maior número de

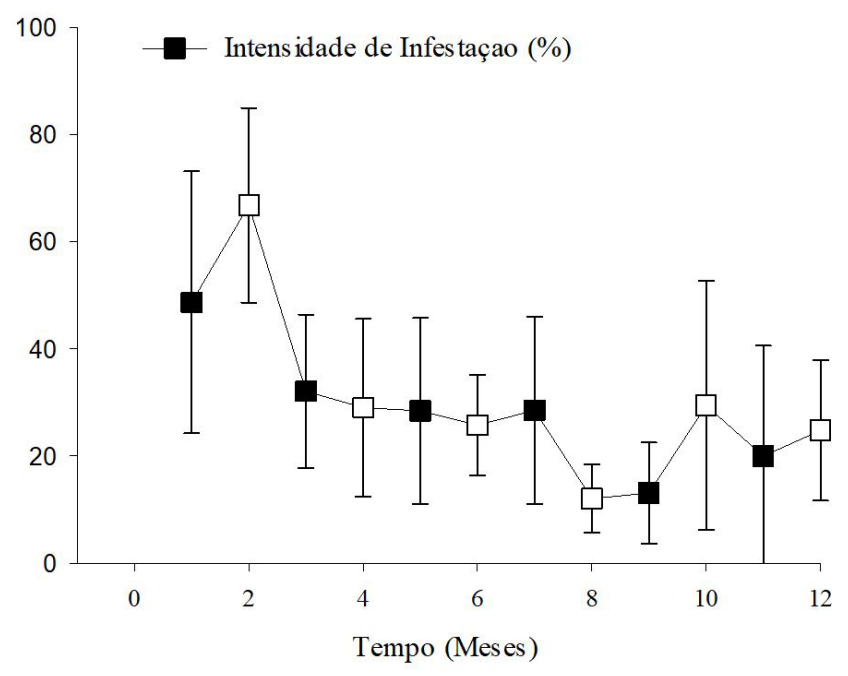

Figura 1. Intensidade de infestação (II) da larva do minador dos citros, em porcentagem (\%), em novas brotações de laranjeiras De Russas, ao longo de 12 meses de avaliação. Russas - CE, Brasil. 2019. As barras no gráfico representam o erro padrão.

Tabela 1. Análise de variância (ANOVA) para intensidade de infestação (II) do minador dos citros, além do número de minas (MF), larvas (LF) e pupas por folhas (PF) de laranjeiras De Russas, ao longo de 12 meses de avaliação. Russas - CE, Brasil. 2019

\begin{tabular}{lccccc}
\hline \multirow{2}{*}{$\begin{array}{l}\text { Fonte de } \\
\text { variação }\end{array}$} & \multirow{2}{*}{ GL } & \multicolumn{4}{c}{ Quadrados médios } \\
\cline { 3 - 5 } & & II (\%) & MF & LF & PF \\
\hline Tratamentos & 11 & $5381,82^{* *}$ & $0,864^{* *}$ & $0,154^{* *}$ & $0,075^{* *}$ \\
Blocos & 5 & $4107,18^{* *}$ & $0,285^{* *}$ & $0,128^{* *}$ & $0,064^{* *}$ \\
\hline Resíduo & 271 & 364,16 & 0,038 & 0,016 & 0,010 \\
C.V. $(\%)$ & & 63,87 & 62,22 & 77,24 & 70,46 \\
\hline
\end{tabular}

**significativo ao nível de $1 \%$ de probabilidade. $\mathrm{GL}=$ graus de liberdade. C.V. $(\%)=$ coeficiente de variação. 
Tabela 2. Comparação de médias dos resultados de brotações com minas (BM) e número de folhas da brotação com minas (FM), além do número de minas (MF), de larvas (LF) e de pupas por folha (PF) em laranjeiras De Russas, ao longo de 12 meses de avaliação. Russas - CE, Brasil. 2019

\begin{tabular}{lccccc}
\hline \multicolumn{1}{c}{ Mês } & BM & FM & MF & LF & PF \\
\hline Janeiro & $1,083 \mathrm{a}$ & $3,188 \mathrm{~b}$ & $0,454 \mathrm{~b}$ & $0,179 \mathrm{c}$ & $0,108 \mathrm{c}$ \\
Fevereiro & $1,373 \mathrm{a}$ & $5,350 \mathrm{a}$ & $0,838 \mathrm{a}$ & $0,356 \mathrm{a}$ & $0,248 \mathrm{a}$ \\
Março & $0,613 \mathrm{~b}$ & $1,471 \mathrm{c}$ & $0,365 \mathrm{~b}$ & $0,183 \mathrm{c}$ & $0,033 \mathrm{c}$ \\
Abril & $0,671 \mathrm{~b}$ & $0,967 \mathrm{c}$ & $0,367 \mathrm{~b}$ & $0,231 \mathrm{~b}$ & $0,154 \mathrm{~b}$ \\
Maio & $0,352 \mathrm{c}$ & $0,554 \mathrm{c}$ & $0,221 \mathrm{c}$ & $0,148 \mathrm{c}$ & $0,071 \mathrm{c}$ \\
Junho & $0,758 \mathrm{~b}$ & $0,817 \mathrm{c}$ & $0,335 \mathrm{~b}$ & $0,231 \mathrm{~b}$ & $0,119 \mathrm{c}$ \\
Julho & $0,598 \mathrm{~b}$ & $0,640 \mathrm{c}$ & $0,252 \mathrm{c}$ & $0,156 \mathrm{c}$ & $0,092 \mathrm{c}$ \\
Agosto & $0,317 \mathrm{c}$ & $0,369 \mathrm{c}$ & $0,196 \mathrm{c}$ & $0,104 \mathrm{~d}$ & $0,056 \mathrm{c}$ \\
Setembro & $0,350 \mathrm{c}$ & $0,460 \mathrm{c}$ & $0,221 \mathrm{c}$ & $0,094 \mathrm{~d}$ & $0,088 \mathrm{c}$ \\
Outubro & $0,252 \mathrm{c}$ & $0,456 \mathrm{c}$ & $0,185 \mathrm{c}$ & $0,106 \mathrm{~d}$ & $0,096 \mathrm{c}$ \\
Novembro & $0,471 \mathrm{c}$ & $0,456 \mathrm{c}$ & $0,196 \mathrm{c}$ & $0,110 \mathrm{~d}$ & $0,090 \mathrm{c}$ \\
Dezembro & $0,210 \mathrm{c}$ & $0,373 \mathrm{c}$ & $0,138 \mathrm{c}$ & $0,069 \mathrm{~d}$ & $0,058 \mathrm{c}$ \\
C.V. $(\%)$ & 71,48 & 84,42 & 62,22 & 77,24 & 70,67 \\
Valor de F & $10,226^{* *}$ & $26,489 * *$ & $22,668^{* *}$ & $9,610^{* *}$ & $7,309^{* *}$ \\
Valor $p$ & 0,0000 & 0,0000 & 0,0000 & 0,0000 & 0,0000 \\
\hline
\end{tabular}

**significativo ao nível de $1 \%$ de probabilidade. As médias seguidas pela mesma letra minúscula na coluna não diferem estatisticamente entre si pelo teste de teste de $\operatorname{Scott} \operatorname{Knott}(p \leq 0,05)$. C.V. $(\%)=$ coeficiente de variação em porcentagem.

minas por folha entre os meses de janeiro a maio, quando às laranjeiras lançaram novas brotações de verão e outono.

A maior intensidade de infestação, além de incidir no número de brotações e de folhas atacadas, que por sua vez indica o aproveitamento do recurso disponível, ou seja, o aumento da oviposição do minador diante do maior número de folhas e ramos com tecido tenro, também resultou em maior número de minas, larvas e pupas por folha, que se associam, possivelmente, a sobreposição de oviposições, e até ciclos, uma vez que a maior parcela das brotações já continham ovos ou larvas. Sendo variáveis responsáveis por indicar o grau de severidade do ataque da praga (Cônsoli et al., 1996; Gravena, 2011).

A ausência de métodos de manejo, e/ou controle, permitiu que as larvas do minador dos citros se alimentassem e desenvolvessem plenamente, formando pupas nas periferias das folhas, praticamente em todos os meses do ano, (Cônsoli et al., 1996; Gravena, 2011; Fundecitrus, 2019). O maior número de pupas foi observado em fevereiro, que diferiu estatisticamente dos demais meses do ano (Tabela 2). O número de pupas por folha variou entre 0,033 (março) e 0,248 (fevereiro) (Tabela 2), corroborando com valores obtidos por Efrom et al., (2006), que estudaram a dinâmica populacional do minador, assim como de seus parasitoides, em pomares de tangerineira 'Montenegrina' (Citrus deliciosa Ten.) e tangoreiro híbrido 'Murcott' (C. sinensis $\times$ Citrus reticulata Blanco), e relacionaram as maiores densidades de pupas ao período de verão e outono, quando as larvas puderam alimentar-se e progredir nos instares até a fase pupal.

Diante dos resultados das principais variáveis (minas, larvas e pupas por folha), se pode constatar que a ocorrência e a sazonalidade do minador dos citros, ampararam-se principalmente no desenvolvimento vegetativo das laranjeiras De Russas, que apesar das variações, forneceram sempre hospedeiro e alimento ao minador, a medida que emitiram novas brotações ao longo dos doze meses de avaliação (Figura 2).

A emissão de novas brotações no período seco deve-se basicamente a adoção de sistemas de irrigação para suprimento hídrico, além de tratos culturais, como poda e indução floral. As plantas cítricas se desenvolvem continuamente desde o transplantio das mudas, quando se adaptam as condições de campo, e beneficiam-se de condições favoráveis para expandir sua capacidade fotossintética, e crescer em altura e diâmetro ao longo da fase juvenil (Mattos Junior et al., 2005; Sombra et al., 2019).

No presente estudo, verificou-se que condições climáticas influenciaram a dinâmica anual das laranjeiras, constatando-se por exemplo, crescimento no número de novas brotações nos quatro primeiros meses (janeiro à abril) (Figura 2), que compõem a estação chuvosa, e caracterizam-se pela mudança entre o clima seco e 


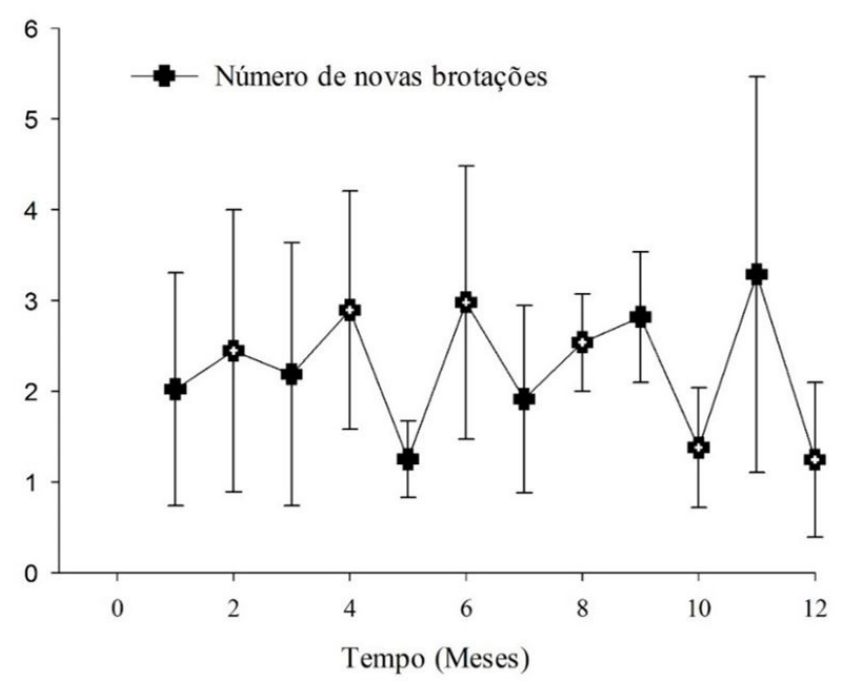

Figura 2. Número de novas brotações em laranjeiras De Russas, ao longo de 12 meses de avaliação. Russas - CE, Brasil. 2019. As barras no gráfico representam o erro padrão.

Tabela 3. Equações da análise de regressão entre temperaturas média, máxima e mínima e a intensidade de infestação (II) em novas brotações de laranja De Russas, Russas - CE, Brasil. 2019

\begin{tabular}{llc}
\hline \multicolumn{1}{c}{$\mathrm{T}\left({ }^{\circ} \mathrm{C}\right)^{1}$} & \multicolumn{1}{c}{ Equação de regressão } & $\mathrm{R}^{2}$ \\
\hline Média & $\mathrm{y}=9.7146 \mathrm{x}^{2}-562.63 \mathrm{x}+8172.9$ & 0.01 \\
Máxima & $\mathrm{y}=-2.0679 \mathrm{x}^{2}+149.96 \mathrm{x}-2686$ & 0.05 \\
Mínima & $\mathrm{y}=-0.7716 \mathrm{x}^{2}+40.271 \mathrm{x}-482.06$ & 0.27 \\
\hline
\end{tabular}

${ }^{1}$ Temperatura do ar. $\mathrm{T}\left({ }^{\circ} \mathrm{C}\right)=$ temperatura do ar em graus celsius. R2 = coeficiente de determinação.

quente, dos últimos meses do ano anterior (setembro à dezembro), e o aumento na frequência de precipitações pluviométricas, que possivelmente, contribuem para o suprimento hídrico e a absorção de nutrientes na cultura, além de amenizar o clima, reduzindo a temperatura e favorecendo a umidade relativa do ar (Gheyi et al., 2012; Alvares et al., 2013).

As novas brotações, que apresentavam menor incidência de injúrias do minador dos citros, se desenvolveram e amadureceram ao longo dos meses, permitindo as laranjeiras crescer em altura e diâmetro (Figura 3), e consequentemente, expandir a atividade fotossintética no período seco (julho à dezembro), quando o fotoperíodo, a irradiação solar e as temperaturas foram mais elevadas, potencializando o metabolismo vegetal (Mattos Junior et al., 2005; Gheyi et al., 2012).

No entanto, com o desenvolvimento das folhas, que ganharam tamanho e espessura, e tornaram-se mais resistentes, constatou-se redução na densidade populacional do minador dos citros (Tabela 2), possivelmente devido

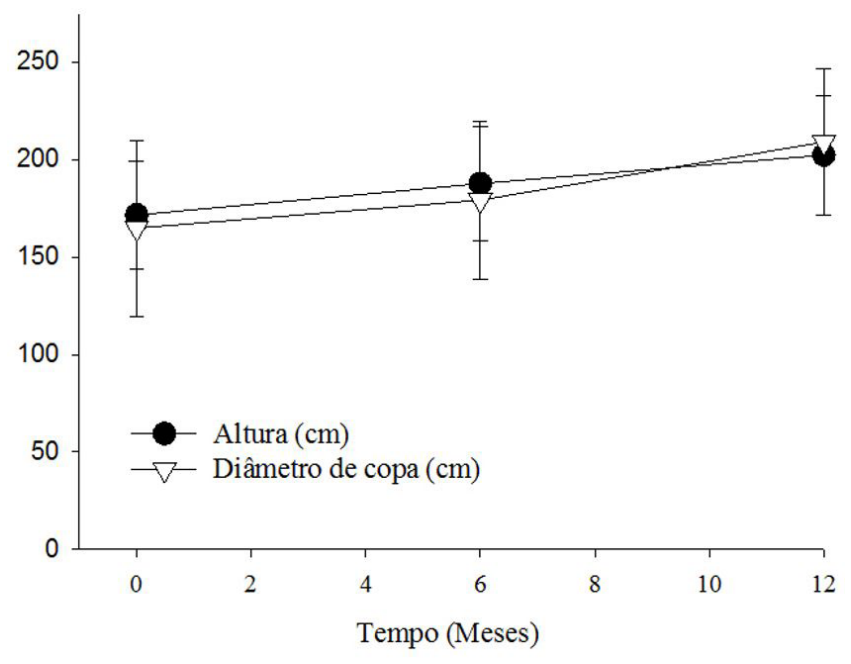

Figura 3. Altura (H) e diâmetro médio de copa (D) de laranjeiras De Russas, em centímetros (cm), ao longo de 12 meses de avaliação. Russas - CE, Brasil. 2019. As barras no gráfico representam o erro padrão.

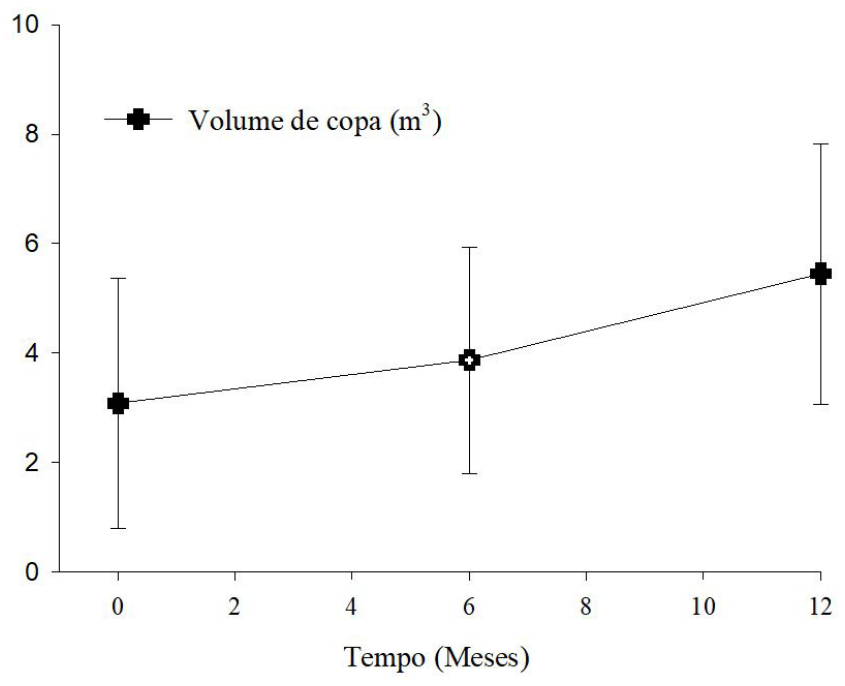

Figura 4. Volume médio de copa $\left(\mathrm{V}^{3}\right)$ de laranjeiras De Russas, em metros cúbicos $\left(\mathrm{m}^{3}\right)$ ao longo de 12 meses de avaliação. Russas - CE, Brasil. 2019. As barras no gráfico representam o erro padrão.

a menor preferência de oviposição do micro lepidóptero por este hospedeiro, geralmente associada também a não preferência alimentar das larvas (Cônsoli et al., 1996; Brito et al., 2017). Os valores de altura e diâmetro incidiram na conformação da copa, inferindo crescimento no volume das laranjeiras De Russas, que se acentuou no segundo semestre (Figura 4).

A estimativa destes parâmetros pode permitir a associação entre a ocorrência do minador, com o desenvolvimento vegetativo e as condições climáticas. Principalmente, ao considerar que as fêmeas do minador ovipositam 
preferencialmente em tecidos foliares tenros, como novas brotações, emitidas sazonalmente à medida que as condições climáticas se alteram, ou mesmo, pela realização, ou não, de tratos cultura, como podas (Yamamoto, 2008; Gravena, 2011; Fundecitrus, 2019). Presumindo-se, que o porte da planta se associa a sua capacidade de emissão de brotações favoráveis à reprodução da praga, o que dificulta seu controle (Cunha Sobrinho et al., 2013; Scarpin, 2014).

A temperatura média variou principalmente entre os meses de janeiro e março, crescendo de $27,02{ }^{\circ} \mathrm{C}$ para $29,63{ }^{\circ} \mathrm{C}$ (Figura 5), possivelmente, devido à estiagem registrada entre janeiro e fevereiro. Após, a temperado decresceu e regularizou-se a medida que as precipitações se intensificaram (Figura 6), levando ao registro dos menores valores até o mês de agosto. Já ao considerar o intervalor entre as temperaturas máximas e mínimas, constata-se a máxima de $38,6{ }^{\circ} \mathrm{C}$ ocorreu no mês de outubro, e a mínima de $19,8{ }^{\circ} \mathrm{C}$ em julho (Figura 5).

A variação sazonal observada é característica, e geralmente, determina o regime térmico e pluviométrico predominante na região semiárida, uma vez que o clima é um condicionante do meio ambiente, capaz de atuar sobre a dinâmica dos agroecossistemas e interferir no metabolismo vegetal, restringindo ou potencializando a emissão e o desenvolvimento de novas brotações (Mattos Junior et al., 2005; Gheyi et al., 2012). Apesar da sensibilidade dos insetos as condições climáticas (pecilotérmicos), não se registrou correlação com a intensidade de infestação do minador dos citros, constatando-se coeficientes de regressão $\left(\mathrm{R}^{2}\right)$ considerados baixos (Tabela 3 ).

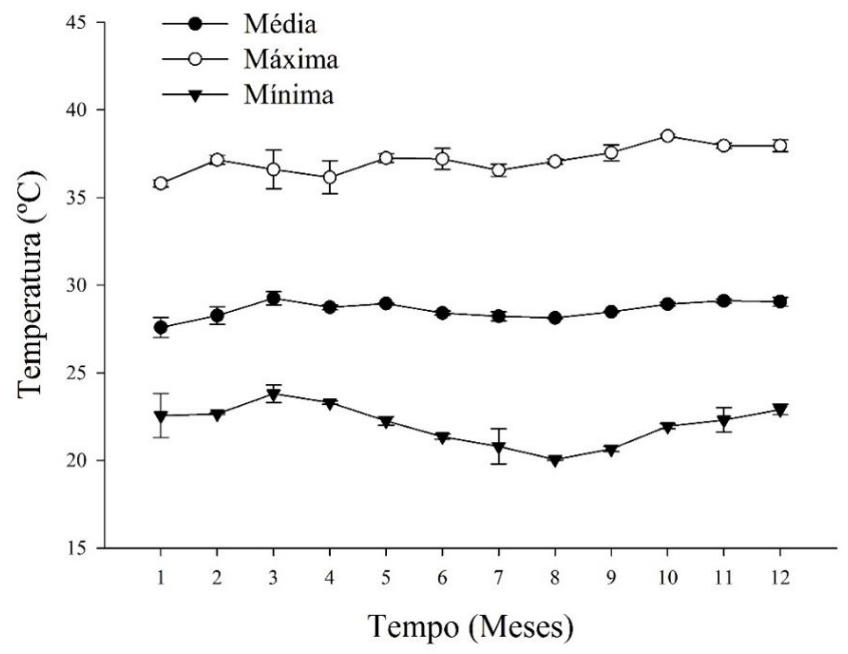

Figura 5. Valores de temperatura média, máxima e mínima, registradas pela estação automática do INMET, Posto Jaguaruana, ao longo de 12 meses de avaliação. Russas - CE. Brasil. 2019. As barras no gráfico representam o erro padrão.
Na Índia, Patel \& Patel (2001) constataram que as temperaturas máximas e mínimas, além da umidade relativa do ar, foram os fatores de maior impacto na flutuação populacional do minador dos citros em pomares de limeira "Kagzi" (Citrus Aurantifolia (Christm.) Swingle). Ao considerar que os insetos são organismos pecilotérmicos, se pode inferir que a temperatura é condicionante de sua fisiologia, e consequentemente, de seu comportamento alimentar e reprodutivo, como se constata por exemplo, na ampla variação de duração do ciclo de vida do minador dos citros, que em temperaturas próximas a $32{ }^{\circ} \mathrm{C}$ pode durar apenas 11,5 dias (ovo-ovo). No entanto, em temperaturas mais baixas, próximas de $18{ }^{\circ} \mathrm{C}$, o ciclo pode chegar a 32,7 dias, de acordo com a qualidade do alimento para o desenvolvimento larval, principal fase do inseto, e que é afetada pela variedade de citros (Chagas \& Parra, 2000).

Considerando a precipitação pluviométrica durante os 12 meses de avaliação no município de Russas, o volume acumulado foi de $605 \mathrm{~mm}$, abaixo da média histórica dos últimos 40 anos, de $750 \mathrm{~mm}$ (INMET, 2018). As precipitações concentraram-se entre os meses de janeiro e fevereiro, meses em que se concentraram as emissões de brotações, e consequentemente, maior intensidade de infestação do minador dos citros, constatando-se a maior precipitação no mês de fevereiro (195 mm), enquanto não foram registradas precipitações entre julho e novembro (Figura 6).

As precipitações tiveram relação positiva sobre a intensidade de infestação do minador dos citros, atingindo coeficiente de regressão de 0.79 (Tabela 4).

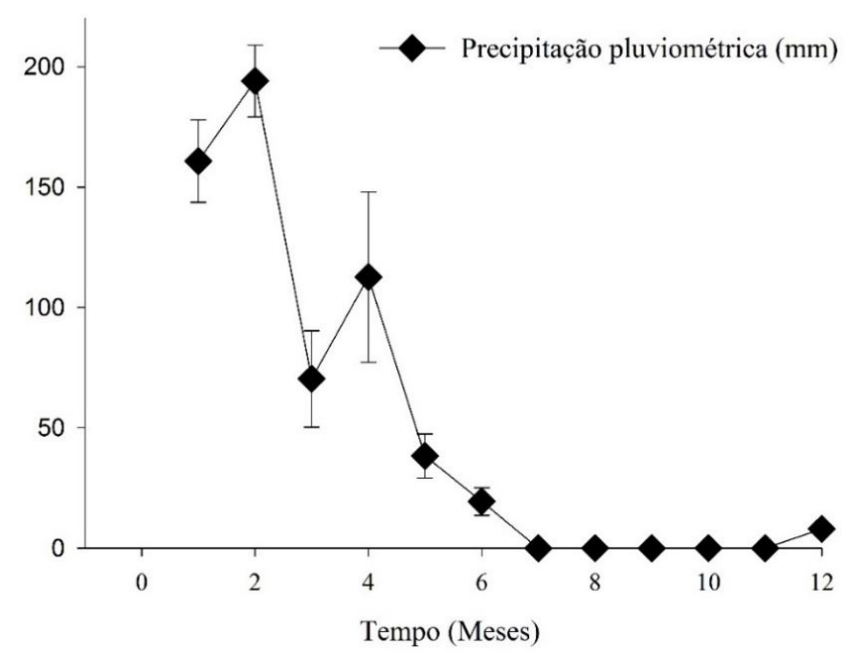

Figura 6. Valores de precipitação pluviométrica pela estação automática do INMET, Posto Jaguaruana, ao longo de 12 meses de avaliação, Russas - CE. Brasil. 2019. As barras no gráfico representam o erro padrão. 
Tabela 4. Equação da análise de regressão entre as precipitações e a intensidade de infestação (II) em novas brotações de laranja De Russas, Russas - CE, Brasil. 2019

\begin{tabular}{ccc}
\hline \multirow{2}{*}{ Precipitação $(\mathrm{mm})$} & Equação de regressão & $\mathrm{R}^{2}$ \\
\cline { 2 - 3 } & $\mathrm{y}=0.0011 \mathrm{x}^{2}+0.3395 \mathrm{x}+21.564$ & 0.79 \\
\hline
\end{tabular}

$\mathrm{R}^{2}=$ coeficiente de determinação.

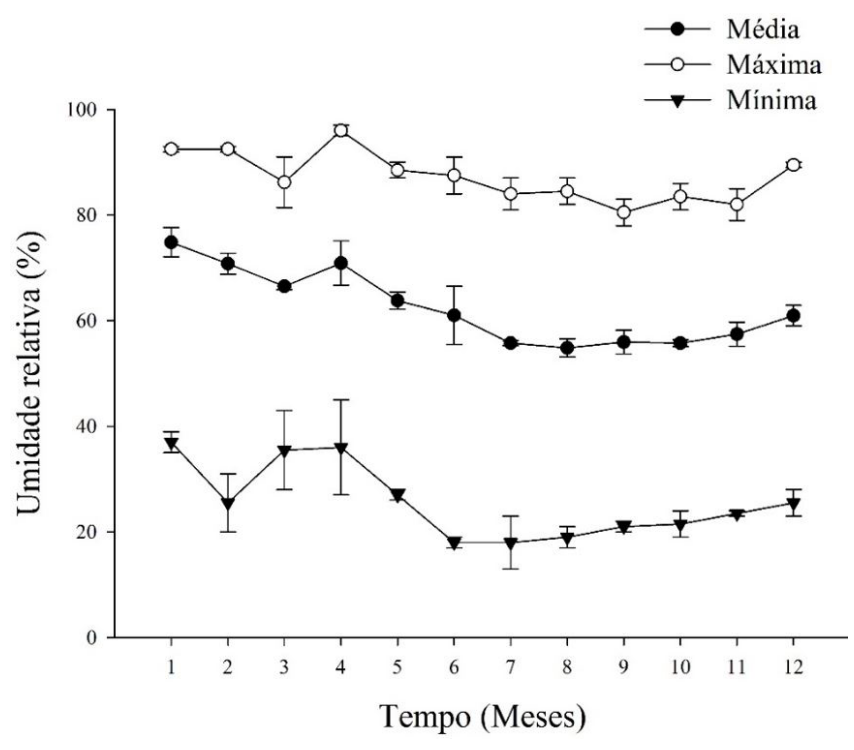

Figura 7. Valores de umidade relativa do ar pela estação automática do INMET, Posto Jaguaruana, ao longo de 12 meses de avaliação, Russas - CE. Brasil. 2019. As barras no gráfico representam o erro padrão.

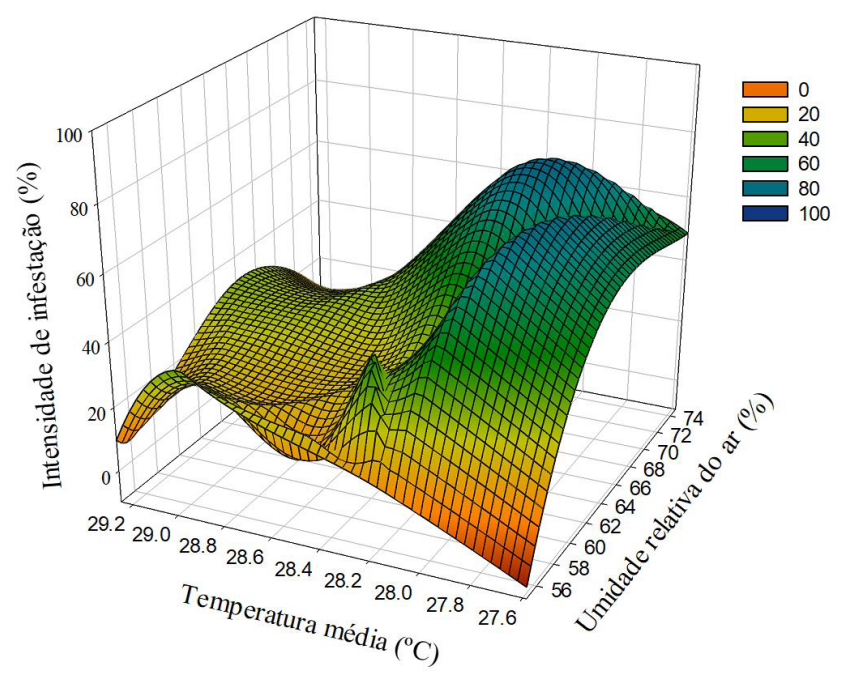

Figura 8. Gráfico superfície de resposta da intensidade de infestação (II), em função da umidade e temperatura do ar, em laranjeiras De Russas. Russas - CE. Brasil. 2019.

Logicamente, esta relação pode ser atribuída a amenização do clima quente e seco, à medida que as precipitações pluviométricas reduziram a temperatura média (Figura 5) e a umidade relativa do ar (Figura 7), favorecendo a emissão de novas brotações e a atuação do microlepidóptero, o que elevou a intensidade de infestação. Corroborando com Jesus et al. (2008), que não registraram a presença de minas quando houve redução no número e na intensidade das precipitações.

A importância da umidade relativa do ar tornou-se evidente com a geração do gráfico de superfície de resposta usando também os valores de temperatura média e intensidade de infestação (Figura 8). Constatando-se que temperaturas amenas aliadas à alta umidade (acima de $60 \%$ ) favoreceram percentuais de infestação acima dos $40 \%$, nível de controle adotado para pomares adultos. Em contrapartida, o aumento da temperatura reduziu a umidade relativa do ar e inibiu o incremento populacional do minador dos citros. Permitindo inferir que valores de temperatura média entre $26,8^{\circ} \mathrm{C}$ e $28,6^{\circ} \mathrm{C}$, e de umidade relativa acima de $60 \%$, são favoráveis ao desenvolvimento do minador-dos-citros.

Ao considerar todos os resultados, é possível verificar que as variações populacionais dos estágios imaturos do minador dos citros, de acordo com Cônsoli (2001), devem-se principalmente a alterações na temperatura, umidade relativa do ar e/ou precipitação pluviométrica, aliadas à presença de brotações novas com tecidos meristemáticos tenros, que permitem a oviposição e o desenvolvimento do minador. Diante disto, pode-se concluir que as edafoclimáticas do semiárido cearense mostraram-se relevantes a ocorrência e a sazonalidade do minador, destacando-se as precipitações como fator mais importante na intensidade de infestação. Os resultados corroboram com Auerbach et al., (1995), que sugeriram que menores precipitações, e consequentemente, menores valores de umidade relativa, reduziam a atividade reprodutiva dos adultos do minador dos citros, visto que esses fatores também interferem sobre a capacidade de voo, seja para dispersão ou oviposição, além de, em chuvas intensas, ocasionarem a exposição das larvas e o desalojamento dos ovos.

Compreender a dinâmica populacional e a sazonalidade do minador dos citros é de extrema relevância para o MIP nos citros, principalmente para auxiliar na mitigação dos riscos de disseminação do cancro cítrico, causado pela 
bactéria Xanthomonas axonopodis pv. citri. (Figueiredo Neto et al., 2006). Sendo assim, a produção de laranja 'De Russas' na microrregião do Baixo Jaguaribe, em condições edafoclimáticas similares, pode considerar a predominância de ocorrência do minador dos citros nos primeiros meses do ano, principalmente entre janeiro e abril, com atenção à possíveis picos populacionais em fevereiro, diante das condições climáticas favoráveis, com maior número e intensidade de precipitações, que reduzem a temperatura e melhoram a umidade relativa do ar, em condições semiáridas.

\section{CONCLUSÕES}

A oferta de alimento (novas brotações) para as larvas ao longo do ano, apesar de influenciar na ocorrência do minador, não foi o fator mais significativo, verificando-se que a densidade populacional variou principalmente devido a temperatura e umidade. Os maiores índices de larvas e pupas foram registrados nos primeiros meses do ano, resultando em $43 \%$ de brotações atacadas e até 3 minas por folha. Destacando-se as condições climáticas com temperaturas próximas à $26^{\circ} \mathrm{C}$ e umidade relativa de $60 \%$.

Conhecer a dinâmica populacional do minador dos citros, assim como, as condições mais favoráveis ao seu surgimento, podem otimizar o manejo integrado de pragas, permitindo a adoção de técnicas preventivas para manter as populações de minador dos citros abaixo do nível de controle.

\section{REFERÊNCIAS}

Alvares, C. A., Stape, J. L., Sentelhas, P. C., De Moraes, G., Leonardo, J., \& Sparovek, G. (2013). Köppen's climate classification map for Brazil. Meteorologische Zeitschrift, 22(6), 711-728.

Arshad, M., Ullah, M. I., Qureshi, J. A., \& Afzal, M. (2018). Physiological effects of citrus leafminer Phyllocnistis citrella (Lepidoptera: Gracillariidae) Larval feeding on photosynthetic and gaseous exchange rates in citrus. Journal of Economic Entomology, 111(5), 2264-2271.

Auerbach, M. J., Connor, E. F., \& Mopper, S. (1995). Minor miners and major miners: population dynamics of leaf-mining insects. In N. Cappuccino, \& P. W. Price (Eds.), Population dynamics (p. 83-100). London: Academic Press.
Bastos, D. C., Sombra, K. E. S., Andrade, H. M., Santos Filho, L. G., \& Passos, O. S. (2017a). Biometric evaluation of orange cultivars using different rootstocks in the semiarid region of Ceará, Brazil. Citrus Research \& Technology, 38(1), 71-76.

Bastos, D. C., Sombra, K. E. S., Loureiro, F. L. C., Silva, A. C. C., \& Passos, O. S. (2017b). Initial development of Tahiti acid lime trees on different rootstocks in the semiarid region of Ceará, Brazil. Citrus Research \& Technology, 38(1), 77-82.

Brito, R., Lopez-Vaamonde, C., Goncalves, G. L., Becker, V. O., Mielke, O. H., \& Moreira, G. R. (2017). Taxonomic revision of Neotropical Phyllocnistis Zeller, 1848 (Lepidoptera: Gracillariidae), with descriptions of seven new species and host plant associations. Zootaxa, 4341(3), 301-352.

Chagas, M. C. M., \& Parra, J. R. P. (2000). Phyllocnistis citrella Stainton (Lepidoptera: Gracillariidae): técnica de criação e biologia em diferentes temperaturas. Anais da Sociedade Entomológica do Brasil, 29(1), 227-235.

Cônsoli, F. L. (2001). Lagarta-minadora-dos-citros, Phyllocnistis citrella (Lepidoptera: Gracillariidae). In E. Vilela, R. A. Zucchi, \& F. Cantor (Eds.), Histórico e impacto das pragas introduzidas no Brasil (pp. 23-30). Ribeirão Preto: Holos.

Cônsoli, F. L., Zucchi, R. A., \& Lopes, J. R. S. (1996). A lagarta minadora dos citros. Piracicaba: Fealq.

Cunha Sobrinho, A. P., Magalhães, A. F. J., Souza, A. S., Passos, O. S., \& Soares Filho, W. S. (2013). Cultura do citros. Brasília: Embrapa Informação Tecnológica.

De Morais, M. R., Zanardi, O. Z., Rugno, G. R., \& Yamamoto, P. T. (2016). Impact of five insecticides used to control citrus pests on the parasitoid Ageniaspis citricola Longvinovskaya (Hymenoptera: Encyrtidae). Ecotoxicology, 25(5), 1011-1020.

Efrom, C. F. S., Redaelli, L. R., \& Diefenbach, L. M. G. (2006). Variação populacional de Phyllocnistis citrella e parasitismo em variedades de citros sob manejo orgânico. Manejo Integrado de Plagas y Agroecología, 78(1), 46-55.

Ferreira, D. F. (2014). Sisvar: a guide for its bootstrap procedures in multiple comparisons. Ciência e Agrotecnologia, 38(2), 109-112.

Figueiredo Neto, E., Lopes, M. P. C., Palharin, L. H. D. C., \& Sambugaro, R. (2006). Revisão literária sobre 
cancro cítrico (Xanthomonas axonopodis pv. citri). Revista Científica Eletrônica de Agronomia, 5(10), 1-7.

Fundo de Defesa da Citricultura - Fundecitrus. (2019). Minador dos citros. Recuperado em 30 de janeiro de 2019, de http://www.fundecitrus.com.br/doencas/minador/8

Garijo, C., \& García, E. J. (1994). Phyllocnistis citrella (Stainton, 1856) (Insecta: Lepidoptera: Gracillariidae: Phyllocnistinae) en los cultivos de cítricos de Andalucía (Sur España): biología, ecología y control de la plaga. Boletin de Sanidad Vegetal: Plagas, 20(4), 815-826.

Gheyi, H. R., Paz, V. P. S., Medeiros, S. S., \& Galvão, C. O. (2012). Recursos hídricos em regiões semiáridas. Campina Grande: Instituto Nacional do Semiárido. Cruz das Almas: Universidade Federal do Recôncavo da Bahia.

Gravena, S. (2005). Manual prático de manejo ecológico de pragas dos citros. Jaboticabal: Gravena.

Gravena, S. (2011). História do controle de pragas na citricultura brasileira. Citrus Research \& Technology, 32(2), 85-92.

Greve, C., \& Redaelli, L. R. (2006). Variação sazonal dos estágios imaturos de Phyllocnistis citrella Stainton (Lepidoptera: Gracillariidae) em pomares de Citrus sinensis sob dois sistemas de cultivo. Neotropical Entomology, $35(6), 828-833$.

Instituto Nacional de Meteorologia - INMET. (2018). Banco de Dados Meteorológicos para Ensino e Pesquisa - BDMEP. Recuperado em 21 de dezembro de 2018, de http://www.inmet.gov.br/portal/index.php?r=bdmep/bdmep/

Jahnke, S. M., Redaelli, L. R., Diefenbach, L. M. G., \& Efrom, C. F. (2008). Spatial distribution of parasitism on Phyllocnistis citrella Stainton, 1856 (Lepidoptera: Gracillariidae) in citrus orchards. Brazilian Journal of Biology = Revista Brasileira de Biologia, 68(4), 813-817.

Jesus, C. R., Redaelli, L. R., \& Soglio, F. K. D. (2008). Flutuação populacional de Phyllocnistis citrella Stainton em Citrus deliciosa e no híbrido Murcott Citrus sinensis $x$ Citrus reticulata. Ciência Rural, 38(3), 593-600.

Keathley, C. P., Stelinski, L. L., \& Lapointe, S. L. (2015). Investigating dormant-season application of pheromone in citrus to control overwintering and spring populations of Phyllocnistis citrella (Lepidoptera: Gracillariidae). Pest Management Science, 72(7), 1405-1410.

Mattos Junior, D., De Negri, J. D., Pio, R. M., \& Pompeu Junior, J. (2005). Citros. Campinas: Instituto Agronômico e Fundag.
Paiva, P. E. B., \& Yamamoto, P. T. (2015). Natural parasitism of the citrus leafminer (Lepidoptera: Gracillariidae) over eight years in seven citrus regions of São Paulo, Brazil. The Florida Entomologist, 98(2), 660-665.

Paleari, L. M., Ferraz, J. M. G., Fagundes, G., \& Habib, M. (2001). Novo método de amostragem para monitoramento de Phyllocnistis citrella Stainton, 1856, e de seus inimigos naturais. Laranja, 22(2), 333-349.

Parra, J. R. P., Bento, J. M. S., Chagas, M. C. M., \& Yamamoto, P. T. (2004). O controle biológico da larvaminadora-dos-citros. Visão Agrícola, 1(2), 64-67.

Patel, G. P., \& Patel, J. R. (2001). Population dynamics of Phyllocnistis citrella on citrus in middle Gujarat. Indian Journal of Entomology, 63(1), 41-48.

Peña, J. E., \& Schaffer, B. (1997). Intraplant distribution and sampling of the citrus leafminer (Lepidoptera: Gracillariidae) on lime. Journal of Economic Entomology, 90(2), 458-464.

Pereira, L. G. B. (2008). Minador-das-folhas-dos-citros e bicho furão: pragas de importância econômica da cultura do citrus sp. Belo Horizonte: CETEC, Fundação Centro Tecnológico de Minas Gerais.

Qureshi, J. A., Kostyk, B. C., \& Stansly, P. A. (2017). Single and multiple modes of action insecticides for control of asian citrus psyllid and citrus leafminer. HortScience, 52(5), 732-735.

Rossetti, V. V. (2001). Manual ilustrado de doenças dos citros. Piracicaba: Fealq/Fundecitrus.

Scarpin, M. S. (2014). Adequação de volume de calda e dose de bactericida cúprico para o controle de cancro cítrico (Dissertação de mestrado). Fundo de Defesa da Citricultura, Araraquara, SP.

Sombra, K. E. S., Andrade, H. M., Loureiro, F. L. C., Oliveira, F. E., Bastos, D. C., \& Uchôa, C. N. (2019). Desenvolvimento de laranjeiras variedade de Russas pés francos e clones enxertados no semiárido Cearense. Nativa, 7(1), 6-12.

Sombra, K. E. S., Loureiro, F. L. C., Silva, A. C. C., Santos Filho, L. G. D., Silva, J. A. N., \& Uchoa, C. N. (2018c). Atributos físicos e químicos de solos de pomares de laranja doce no semiárido do Ceará, Brasil. Citrus Research \& Technology, 39, e1041.

Sombra, K. E. S., Loureiro, F. L. C., Silva, A. C. C., Sombra Júnior, C. A., Passos, O. S., \& Bastos, D. C. (2017) Desenvolvimento inicial de Tangerineira-Tangor 
Piemonte sobre diferentes porta-enxertos no Semiárido. In A. T. S. Alfaro, \& D. G. Trojan (Eds.), Descobertas das ciências agrárias e ambientais. Ponta Grossa: Atena.

Sombra, K. E. S., Silva, A. C. C., Loureiro, F. L. C., \& Bastos, D. C. (2016). Citricultura desenvolvida na agricultura de base familiar do município de Russas, Ceará. Revista Cultura Agronômica, 25(3), 303-316.

Sombra, K. E. S., Silva, A. C. C., Loureiro, F. L. C., \& Uchôa, C. N. A. (2018a). Citricultura como Instrumento de Preservação da Agricultura Familiar no Semiárido Cearense, Brasil. Revista de Extensão e Estudos Rurais, 7(1), 353-372.

Sombra, K. E. S., Loureiro, F. L. C., Silva, A. C. C., Silva, M. P., Passos, O. S., \& Bastos, D. C. (2018b). Avaliação biométrica de tangelo Page e pomelo Flame sobre diferentes porta-enxertos no semiárido do Ceará, Brasil. Citrus Research \& Technology, 39, e-1038.

Turrell, F. M. (1946). Tables of surfaces and volumes of spheres and of prolate and oblate spheroids and spheroidal coefficients. Berkeley: University of California.

Yamamoto, P. T. (Ed.) (2008). Manejo integrado de pragas dos citros. Piracicaba: CP 2.

Recebido: Maio 22, 2019

Aceito: Agosto 08, 2020

Como citar: Loureiro, F. L. C., Silva, R. I. R., Gadelha, P. H. M., Sombra, K. E. S., Passos, O. S., Bastos, D. C., Moura, R. D., \& Uchôa, C. N. (2020). Dinâmica populacional de minador dos citros em pomares de laranja De Russas no semiárido cearense. Citrus Research \& Technology, 41, e1059. https://doi. org/10.4322/crt.20520 